\title{
Convex and monotone operator functions
}

\author{
by Jaspal Singh Aujla (Jalandhar) and \\ H. L. VASudeva (Chandigarh)
}

\begin{abstract}
The purpose of this note is to provide characterizations of operator convexity and give an alternative proof of a two-dimensional analogue of a theorem of Löwner concerning operator monotonicity.
\end{abstract}

1. Introduction. For $m \in \mathbb{N}$, let $M_{m}$ be the algebra of all hermitian $m \times m$ complex matrices. Let $I$ be an interval of $\mathbb{R}$. We denote by $M_{m}(I)$ the set of all members of $M_{m}$ whose spectrum is contained in $I$. Let $f$ be a real function of a real variable $x$ in $I$. Let $A=\sum_{i=1}^{m^{\prime}} \lambda_{i} P_{i}\left(m^{\prime} \leq m\right)$ be the spectral resolution of an $A \in M_{m}(I)$. By $f(A)$ we understand the matrix $f(A)=\sum_{i=1}^{m^{\prime}} f\left(\lambda_{i}\right) P_{i}$.

A real function $f$ on an interval $I$ is operator monotone if for each $m \in \mathbb{N}$ and for every pair $A, B \in M_{m}(I)$ with $A \leq B$, we have $f(A) \leq f(B)$. Likewise we say that $f$ is operator convex if for each $m \in \mathbb{N}, f(t A+$ $(1-t) B) \leq t f(A)+(1-t) f(B)$ for all $A, B \in M_{m}(I)$ and every $t \in[0,1]$.

For known results on operator monotone functions and operator convex functions we refer to Ando [1] and Donoghue [3] rather than to original sources.

Let $m, n \in \mathbb{N}$ and $I, J$ be intervals of $\mathbb{R}$. Let $f$ be a real-valued function of two real variables $x$ in $I$ and $y$ in $J$. Let $A \in M_{m}(I)$ and $B \in M_{n}(J)$ have spectral resolutions $A=\sum_{i=1}^{m^{\prime}} \lambda_{i} P_{i}\left(m^{\prime} \leq m\right)$ and $B=\sum_{j=1}^{n^{\prime}} \mu_{j} Q_{j}\left(n^{\prime} \leq n\right)$. Then $f(A, B)$, as in Korányi [5], is the matrix

$$
f(A, B)=\sum_{i=1}^{m^{\prime}} \sum_{j=1}^{n^{\prime}} f\left(\lambda_{i}, \mu_{j}\right) P_{i} \otimes Q_{j} .
$$

$f$ is called operator monotone on $I \times J$ if for each $m, n \in \mathbb{N}$ and for every $A, A_{1} \in M_{m}(I)$ and $B, B_{1} \in M_{n}(J)$ with $A \leq A_{1}$ and $B \leq B_{1}$ we have the

1991 Mathematics Subject Classification: 15A45, 26B25.

Key words and phrases: operator monotone function, operator convex function. 
inequality

$$
f\left(A_{1}, B_{1}\right)-f\left(A_{1}, B\right)-f\left(A, B_{1}\right)+f(A, B) \geq 0 .
$$

Likewise $f$ is called operator convex on $I \times J$ if for each $m, n \in \mathbb{N}$,

$$
f\left(t(A, B)+(1-t)\left(A_{1}, B_{1}\right)\right) \leq t f(A, B)+(1-t) f\left(A_{1}, B_{1}\right)
$$

for all $A, A_{1} \in M_{m}(I), B, B_{1} \in M_{n}(J)$ and for every $t \in[0,1]$.

It is the purpose of this note to give characterizations of convex operator functions analogous to those of real-valued convex functions of one or more real variables $[8, \mathrm{pp} .98-103]$. In the final section, we also provide an alternative proof of a theorem of Korányi [5, Th. 4].

2. One variable case. Consider an open interval $I$ in $\mathbb{R}$ and a continuously differentiable function $f$ on $I$. Fix $n \in \mathbb{N}$ and take $A \in M_{n}(I)$. If $\left\{e_{i j}: 1 \leq i, j \leq n\right\}$ is a system of matrix units for $M_{n}$ such that $A=\sum \lambda_{i} e_{i i}$, we shall denote by $f^{[1]}(A)$ the element in $M_{n}$ with

$$
\left.f^{[1]}(A)\right|_{i, j}= \begin{cases}\left(\lambda_{i}-\lambda_{j}\right)^{-1}\left(f\left(\lambda_{i}\right)-f\left(\lambda_{j}\right)\right) & \text { if } \lambda_{i} \neq \lambda_{j}, \\ f^{\prime}\left(\lambda_{i}\right) & \text { if } \lambda_{i}=\lambda_{j} .\end{cases}
$$

Recall that the spectral resolution of a matrix in $M_{n}$ yields a system of matrix units and that a system of matrix units yields the Hadamard product operation on matrices. The symbol $\circ_{X}$ shall denote the Hadamard product of matrices in a basis that diagonalizes $X$.

2.1. Lemma [4, Lemma 3.1 or 2, III]. With $f$ and $A$ as above, we have

$$
\lim _{\varepsilon \rightarrow 0} \varepsilon^{-1}\{f(A+\varepsilon H)-f(A)\}=f^{[1]}(A) \circ_{A} H
$$

for every $H \in M_{n}$.

For a proof of the lemma, the reader is referred to [4, Lemma 3.1] or $[2, \mathrm{III}]$.

2.2. TheOREM. For a function $f \in C^{1}(I)$, the following statements are equivalent:

(i) $f$ is operator convex on $I$,

(ii) $f(A)-f(B)-f^{[1]}(B) \circ_{B}(A-B) \geq 0$ for all $A, B \in M_{n}(I)$ and

(iii) $f^{[1]}(A) \circ_{A}(A-B)-f^{[1]}(B) \circ_{B}(A-B) \geq 0$ for all $A, B \in M_{n}(I)$.

Proof. (i) $\Rightarrow$ (ii). Fix $n$ and take $B \in M_{n}(I)$. Choose a system of matrix units for $M_{n}$ that diagonalizes $B$. For $A \in M_{n}(I)$ and $t \in[0,1]$, we have

$$
f(B+t(A-B)) \leq(1-t) f(B)+t f(A) .
$$


This implies

$$
\begin{aligned}
0 & =\lim _{t \rightarrow 0} t^{-1}\left\{f(B+t(A-B))-f(B)-t f^{[1]}(B) \circ_{B}(A-B)\right\} \\
& \leq f(A)-f(B)-f^{[1]}(B) \circ_{B}(A-B),
\end{aligned}
$$

by Lemma 2.1 and the foregoing inequality.

(ii) $\Rightarrow$ (iii). Fix $n \in \mathbb{N}$. Let $A, B \in M_{n}(I)$. Then

$$
f(A)-f(B) \geq f^{[1]}(B) \circ_{B}(A-B)
$$

and

$$
f(B)-f(A) \geq f^{[1]}(A) \circ_{A}(B-A) .
$$

On adding the last two inequalities, one gets

$$
f^{[1]}(A) \circ_{A}(A-B)-f^{[1]}(B) \circ_{B}(A-B) \geq 0 .
$$

(iii) $\Rightarrow\left(\right.$ i). Let $\varphi:[0,1] \rightarrow M_{n}$ be defined by

$$
\varphi(t)=f(t A+(1-t) B), \quad A, B \in M_{n}(I) .
$$

For $0 \leq t_{1}<t_{2} \leq 1$, let $U_{i}=t_{i} A+\left(1-t_{i}\right) B, i=1,2$. Then $U_{2}-U_{1}=$ $\left(t_{2}-t_{1}\right)(A-B)$. In view of the given condition, we have

$$
f^{[1]}\left(U_{2}\right) \circ_{U_{2}}\left(U_{2}-U_{1}\right)-f^{[1]}\left(U_{1}\right) \circ_{U_{1}}\left(U_{2}-U_{1}\right) \geq 0 .
$$

Observe that

$$
\varphi^{\prime}(t)=\lim _{h \rightarrow 0} h^{-1}\{\varphi(t+h)-\varphi(t)\}=f^{[1]}(t A+(1-t) B) \circ_{X}(A-B),
$$

where $X=t A+(1-t) B$, by Lemma 2.1. Now,

$$
\varphi^{\prime}\left(t_{1}\right)=f^{[1]}\left(U_{1}\right) \circ_{U_{1}}\left(U_{2}-U_{1}\right) \leq f^{[1]}\left(U_{2}\right) \circ_{U_{2}}\left(U_{2}-U_{1}\right)=\varphi^{\prime}\left(t_{2}\right),
$$

i.e. $\varphi^{\prime}$ is increasing. Consequently, $\varphi$ is convex. Therefore,

$$
\begin{aligned}
f(t A+(1-t) B) & =\varphi(t)=\varphi(t \cdot 1+(1-t) \cdot 0) \\
& \leq t \varphi(1)+(1-t) \varphi(0)=t f(A)+(1-t) f(B) .
\end{aligned}
$$

For our next result, we need the following lemma.

2.3. Lemma (cf. [2, III]). Let $f \in C^{2}(I)$. Fix $n$ and take $A \in M_{n}(I)$. If $\left\{e_{i j}: 1 \leq i, j \leq n\right\}$ is a system of matrix units for $M_{n}$ such that $A=\sum \lambda_{i} e_{i i}$, then

$$
\begin{aligned}
e_{i i} \lim _{\varepsilon \rightarrow 0} \varepsilon^{-2}\left\{f(A+\varepsilon H)-f(A)-\varepsilon f^{[1]}(A) \circ_{A} H\right\} e_{j j} \\
=\frac{1}{\lambda_{i}-\lambda_{j}} \sum_{k}\left\{\frac{f\left(\lambda_{i}\right)-f\left(\lambda_{k}\right)}{\lambda_{i}-\lambda_{k}}-\frac{f\left(\lambda_{j}\right)-f\left(\lambda_{k}\right)}{\lambda_{j}-\lambda_{k}}\right\} h_{i k} h_{k j} e_{i j}
\end{aligned}
$$

for every $H \in M_{n}$ and for $\lambda_{i} \neq \lambda_{j} \neq \lambda_{k}$. In case $\lambda_{i}=\lambda_{j}$ or $\lambda_{j}=\lambda_{k}$ or $\lambda_{i}=\lambda_{k}$, the difference quotient on the right hand side is to be replaced by the appropriate derivative. 
Proof. If $f(x)=x^{p}$ then the second order term in $\varepsilon$ of $f(A+\varepsilon H)$ is $\sum A^{m} H A^{r} H A^{s}$, the summation being taken over all $m, r, s \geq 0$ such that $m+r+s=p-2$. Consequently,

$$
\begin{aligned}
e_{i i} \sum_{m, r, s} A^{m} H A^{r} H A^{s} e_{j j} & =\sum_{m, r, s} \lambda_{i}^{m} \lambda_{j}^{s} e_{i i} H A^{r} H e_{j j} \\
& =\sum_{m, r, s, k} \lambda_{i}^{m} \lambda_{j}^{s} \lambda_{k}^{r} h_{i k} h_{k j} e_{i j} \\
& =\left(\lambda_{i}-\lambda_{j}\right)^{-1} \sum_{k}\left\{\frac{\lambda_{i}^{p}-\lambda_{k}^{p}}{\lambda_{i}-\lambda_{k}}-\frac{\lambda_{j}^{p}-\lambda_{k}^{p}}{\lambda_{j}-\lambda_{k}}\right\} h_{i k} h_{k j} e_{i j}
\end{aligned}
$$

as desired. Since the linear span of such functions is dense in $C^{2}(I)$ in the topology of uniform convergence on compact sets, the result follows.

2.4. Theorem ([1], Lemma 3.1). If $f \in C^{2}(-1,1)$ and $f(0)=0$, then $f$ is operator convex iff $f^{[2]}(A ; \mu) \geq 0$, where $A=\sum_{i} \lambda_{i} e_{i i}$ and

$$
\left.f^{[2]}(A ; \mu)\right|_{i, j}=\left(\lambda_{i}-\lambda_{j}\right)^{-1}\left\{\frac{f\left(\lambda_{i}\right)-f(\mu)}{\lambda_{i}-\mu}-\frac{f\left(\lambda_{j}\right)-f(\mu)}{\lambda_{j}-\mu}\right\},
$$

and $\mu \in(-1,1)$ is arbitrary. The right hand of the above equality is to be interpreted appropriately in case $\lambda_{i}=\lambda_{j}$ or $\lambda_{i}=\mu$ or $\lambda_{j}=\mu$.

Proof. Fix $n$ and take $A \in M_{n}(I)(I=(-1,1))$ and choose a system of matrix units for $M_{n}$ such that $A$ is diagonal. Let $\mu \in I$ be arbitrary. Let $A^{\prime}=\operatorname{diag}\left(\lambda_{1}, \ldots, \lambda_{n}, \lambda_{n+1}\right)$, where $\lambda_{n+1}=\mu$. Suppose $f$ is operator convex. Then

$$
f\left(A^{\prime}+\delta \varepsilon H\right)=f\left((1-\delta) A^{\prime}+\delta\left(A^{\prime}+\varepsilon H\right)\right) \leq(1-\delta) f\left(A^{\prime}\right)+\delta f\left(A^{\prime}+\varepsilon H\right) .
$$

Dividing by $\delta$ and letting $\delta \rightarrow 0$ gives

$$
0 \leq f\left(A^{\prime}+\varepsilon H\right)-f\left(A^{\prime}\right)-\varepsilon f^{[1]}\left(A^{\prime}\right) \circ_{A} H,
$$

using Lemma 2.1. Lemma 2.3 then implies that the matrix whose $(i, j)$ th entry is

$$
\left(\lambda_{i}-\lambda_{j}\right)^{-1} \sum_{k=1}^{n+1}\left\{\frac{f\left(\lambda_{i}\right)-f\left(\lambda_{k}\right)}{\lambda_{i}-\lambda_{k}}-\frac{f\left(\lambda_{j}\right)-f\left(\lambda_{k}\right)}{\lambda_{j}-\lambda_{k}}\right\} h_{i k} h_{k j}
$$

is non-negative. Choose

$$
H=\left(\begin{array}{ccccc}
0 & 0 & \ldots & 0 & \bar{\xi}_{1} \\
0 & 0 & \ldots & 0 & \bar{\xi}_{2} \\
\ldots & \ldots & \ldots & \ldots & \bar{\xi}_{n} \\
\xi_{1} & \xi_{2} & \ldots & \xi_{n} & 0
\end{array}\right)
$$


Then (1) becomes

$$
\left(\lambda_{i}-\lambda_{j}\right)^{-1}\left\{\frac{f\left(\lambda_{i}\right)-f(\mu)}{\lambda_{i}-\mu}-\frac{f\left(\lambda_{j}\right)-f(\mu)}{\lambda_{j}-\mu}\right\} \bar{\xi}_{i} \xi_{j} .
$$

Since the vector $\left(\xi_{1}, \xi_{2}, \ldots, \xi_{n}\right)$ is arbitrary, it follows that $f^{[2]}(A ; \mu) \geq 0$.

On the other hand, suppose that $f^{[2]}(A ; \mu) \geq 0, \mu \in I$. In particular, $f^{[2]}(A ; 0) \geq 0$, which is $g^{[1]}(A) \geq 0$, where $g(x)=f(x) / x$ for $x \neq 0$ and $g(0)=f^{\prime}(0)$. This implies, by using Lemma 2.1 of [1], that $g(x)$ is operator monotone. Consequently, $f(x)$ is operator convex by Lemma 3.1 of [1].

3. Functions of several variables. In this section, we provide characterizations of operator convexity for functions of two variables.

Consider a real-valued function of two real variables $x$ and $y$ in $I=$ $(-1,1)$. Assume that (i) $f(x, 0)=f(0, y)=0$ for all $x, y$ in $I$ and (ii) the first partial derivatives and the mixed second partial derivative of $f$ exist and are continuous. Fix $m, n \in \mathbb{N}$ and take $A$ in $M_{n}(I)$ and $B$ in $M_{n}(J)$. Let $\left\{e_{i j}: 1 \leq i, j \leq m\right\}$ and $\left\{f_{i j}: 1 \leq i, j \leq n\right\}$ be matrix units for $M_{m}$ and $M_{n}$ respectively such that $A=\sum_{i=1}^{m} \lambda_{i} e_{i i}$ and $B=\sum_{i=1}^{n} \mu_{i} f_{i i}$. Let $H \in M_{m}$ and $K \in M_{n}$ be arbitrary. We shall denote by $f^{[1,0]}(A, B), f^{[0,1]}(A, B)$, $f^{[1,1]}(A, B), f^{[2,0]}(A, B)$ and $f^{[0,2]}(A, B)$ the elements in $M_{m} \otimes M_{n}$ defined by:

$$
\left.f^{[1,0]}(A, B)\right|_{i j ; k l}=\left(\lambda_{i}-\lambda_{k}\right)^{-1}\left\{f\left(\lambda_{i}, \mu_{j}\right)-f\left(\lambda_{k}, \mu_{j}\right)\right\}
$$

if $\lambda_{i} \neq \lambda_{k}$, and equal to $\frac{\partial f}{\partial x}\left(\lambda_{i}, \mu_{j}\right)$ if $\lambda_{i}=\lambda_{k}$;

$$
\left.f^{[0,1]}(A, B)\right|_{i j ; k l}=\left(\mu_{j}-\mu_{l}\right)^{-1}\left\{f\left(\lambda_{i}, \mu_{j}\right)-f\left(\lambda_{i}, \mu_{l}\right)\right\}
$$

if $\mu_{j} \neq \mu_{l}$, and equal to $\frac{\partial f}{\partial y}\left(\lambda_{i}, \mu_{j}\right)$ if $\mu_{j}=\mu_{l}$;

$$
\left.f^{[1,1]}(A, B)\right|_{i j ; k l}=\frac{f\left(\lambda_{i}, \mu_{j}\right)-f\left(\lambda_{i}, \mu_{l}\right)-f\left(\lambda_{k}, \mu_{j}\right)+f\left(\lambda_{k}, \mu_{l}\right)}{\left(\lambda_{i}-\lambda_{k}\right)\left(\mu_{j}-\mu_{l}\right)}
$$

if $\lambda_{i} \neq \lambda_{k}$ and $\mu_{j} \neq \mu_{l}$, and the divided difference is to be interpreted appropriately when $\lambda_{i}=\lambda_{k}$ or $\mu_{j}=\mu_{l}$;

$\left.f^{[2,0]}(A, B) \circ\left(H^{2} \otimes I\right)\right|_{i j ; k l}$

$=\left(\lambda_{i}-\lambda_{k}\right)^{-1} \sum_{\alpha}\left\{\frac{f\left(\lambda_{i}, \mu_{j}\right)-f\left(\lambda_{\alpha}, \mu_{j}\right)}{\lambda_{i}-\lambda_{\alpha}}-\frac{f\left(\lambda_{k}, \mu_{j}\right)-f\left(\lambda_{\alpha}, \mu_{j}\right)}{\lambda_{k}-\lambda_{\alpha}}\right\} h_{i \alpha} h_{\alpha l} \delta_{\alpha l}$

if $\lambda_{i} \neq \lambda_{k}, \lambda_{i} \neq \lambda_{\alpha}, \lambda_{k} \neq \lambda_{\alpha}$, and the divided difference is to be interpreted appropriately when $\lambda_{i}=\lambda_{k}$ or $\lambda_{i}=\lambda_{\alpha}$ or $\lambda_{k}=\lambda_{\alpha}$; finally,

$$
\begin{aligned}
& \left.f^{[0,2]}(A, B) \circ\left(I \otimes K^{2}\right)\right|_{i j ; k l} \\
& =\left(\mu_{j}-\mu_{l}\right)^{-1} \sum_{\alpha}\left\{\frac{f\left(\lambda_{i}, \mu_{j}\right)-f\left(\lambda_{i}, \mu_{\alpha}\right)}{\mu_{j}-\mu_{\alpha}}-\frac{f\left(\lambda_{i}, \mu_{l}\right)-f\left(\lambda_{i}, \mu_{\alpha}\right)}{\mu_{l}-\mu_{\alpha}}\right\} k_{i \alpha} k_{\alpha l} \delta_{i \alpha}
\end{aligned}
$$


if $\mu_{j} \neq \mu_{l}, \mu_{j} \neq \mu_{\alpha}, \mu_{l} \neq \mu_{\alpha}$, and the divided difference is to be interpreted appropriately when $\mu_{j}=\mu_{l}$ or $\mu_{j}=\mu_{\alpha}$ or $\mu_{l}=\mu_{\alpha}$.

If $f(x, y)=g(x) h(y)$, the operator $f(A, B)$ coincides with $g(A) \otimes h(B)$ and the following formulae hold for the Hadamard product $\circ$ of matrices in a basis obtained from the basis that diagonalizes $A$ and $B$ :

$$
\begin{aligned}
f^{[1,0]}(A, B) \circ H \otimes I & =\left(g^{[1]}(A) \circ_{A} H\right) \otimes h(B), \\
f^{[0,1]}(A, B) \circ I \otimes K & =g(A) \otimes\left(h^{[1]}(B) \circ_{B} K\right), \\
f^{[1,1]}(A, B) \circ H \otimes K & =\left(g^{[1]}(A) \circ_{A} H\right) \otimes\left(h^{[1]}(B) \circ_{B} K\right), \\
f^{[2,0]}(A, B) \circ H^{2} \otimes I & =\left(g^{[2]}(A) \circ_{A} H^{2}\right) \otimes h(B), \\
f^{[0,2]}(A, B) \circ I \otimes K^{2} & =g(A) \otimes\left(h^{[2]}(B) \circ_{B} K^{2}\right) .
\end{aligned}
$$

Since every $f$ with the properties stipulated at the beginning of the section is the uniform limit of a sequence of linear combinations of such functions, the following lemma holds:

3.1. Lemma. With $f$ and $A, B$ as above, we have

(i) $\lim _{\varepsilon \rightarrow 0} \varepsilon^{-1}\{f(A+\varepsilon H, B)-f(A, B)\}=f^{[1,0]}(A, B) \circ H \otimes I$,

(ii) $\lim _{\varepsilon \rightarrow 0} \varepsilon^{-1}\{f(A, B+\varepsilon K)-f(A, B)\}=f^{[0,1]}(A, B) \circ I \otimes K$,

(iii) $\lim _{\varepsilon \rightarrow 0} \varepsilon^{-1}\{f(A+\varepsilon H, B+\varepsilon K)-f(A, B)\}=f^{[1,0]}(A, B) \circ H \otimes I$

$$
+f^{[0,1]}(A, B) \circ I \otimes K,
$$

(iv) $\lim _{\substack{\varepsilon_{1} \rightarrow 0 \\ \varepsilon_{2} \rightarrow 0}} \varepsilon_{1}^{-1} \varepsilon_{2}^{-1}\left\{f\left(A+\varepsilon_{1} H, B+\varepsilon_{2} K\right)-f\left(A+\varepsilon_{1} H, B\right)\right.$

$$
\left.-f\left(A, B+\varepsilon_{2} K\right)+f(A, B)\right\}=f^{[1,1]}(A, B) \circ H \otimes K,
$$

(v) $\lim _{\varepsilon \rightarrow 0} \varepsilon^{-2}\left\{f(A+\varepsilon H, B)-f(A, B)-\varepsilon f^{[1,0]}(A, B) \circ H \otimes I\right\}$

$$
=f^{[2,0]}(A, B) \circ H^{2} \otimes I,
$$

(vi) $\lim _{\varepsilon \rightarrow 0} \varepsilon^{-2}\left\{f(A, B+\varepsilon K)-f(A, B)-\varepsilon f^{[0,1]}(A, B) \circ I \otimes K\right\}$

$$
=f^{[0,2]}(A, B) \circ I \otimes K^{2},
$$

for every $H \in M_{m}$ and $K \in M_{n}$.

Remark. $\quad f^{[1,0]}(A, B), \quad f^{[0,1]}(A, B), \quad f^{[1,1]}(A, B), \quad f^{[2,0]}(A, B)$, and $f^{[0,2]}(A, B)$ resemble in many ways the appropriate derivatives of $f$. However, they depend on the basis considered for $M_{m} \otimes M_{n}$.

3.2. THEOREM. Let $f$ be a real-valued function of two real variables $x$ and $y$ in $I=(-1,1)$. Assume that (i) $f(x, 0)=f(0, y)=0$ for all $x, y$ in $I$ and (ii) the first partial derivatives and the mixed second partial derivative of $f$ exist and are continuous. Then the following statements are equivalent:

(i) $f$ is operator convex on $I \times I$, 
(ii) $f(A, B)-f\left(A_{0}, B_{0}\right) \geq f^{[1,0]}\left(A_{0}, B_{0}\right) \circ\left(A-A_{0}\right) \otimes I$

$$
+f^{[0,1]}\left(A_{0}, B_{0}\right) \circ I \otimes\left(B-B_{0}\right)
$$

for all $A_{0}, A \in M_{m}(I), B_{0}, B \in M_{n}(I)$ and for every $m, n \in \mathbb{N}$, and

(iii) $f^{[1,0]}\left(A_{2}, B_{2}\right) \circ\left(A_{2}-A_{1}\right) \otimes I+f^{[0,1]}\left(A_{2}, B_{2}\right) \circ I \otimes\left(B_{2}-B_{1}\right)$

$-f^{[1,0]}\left(A_{1}, B_{1}\right) \circ\left(A_{2}-A_{1}\right) \otimes I-f^{[0,1]}\left(A_{1}, B_{1}\right) \circ I \otimes\left(B_{2}-B_{1}\right) \geq 0$

for all $A_{1}, A_{2}$ in $M_{m}(I)$, all $B_{1}, B_{2}$ in $M_{n}(I)$ and all $m, n \in \mathbb{N}$.

Proof. (i) $\Rightarrow$ (ii). Fix $m$ and $n$ in $\mathbb{N}$ and take $A_{0} \in M_{m}(I)$ and $B_{0} \in$ $M_{n}(I)$. Choose systems of matrix units for $M_{m}$ and $M_{n}$ that diagonalize $A_{0}$ and $B_{0}$. For $A \in M_{m}(I)$ and $B \in M_{n}(I)$ and $t \in[0,1]$, we have

$$
\begin{aligned}
f\left(A_{0}+t\left(A-A_{0}\right), B_{0}+t\left(B-B_{0}\right)\right) & =f\left((1-t)\left(A_{0}, B_{0}\right)+t(A, B)\right) \\
& \leq(1-t) f\left(A_{0}, B_{0}\right)+t f(A, B) .
\end{aligned}
$$

This implies

$\lim _{t \rightarrow 0} t^{-1}\left\{f\left(A_{0}+t\left(A-A_{0}\right), B_{0}+t\left(B-B_{0}\right)\right)-f\left(A_{0}, B_{0}\right)\right\} \leq f(A, B)-f\left(A_{0}, B_{0}\right)$,

i.e.

$f(A, B)-f\left(A_{0}, B_{0}\right) \geq f^{[1,0]}\left(A_{0}, B_{0}\right) \circ\left(A-A_{0}\right) \otimes I+f^{[0,1]}\left(A_{0}, B_{0}\right) \circ I \otimes\left(B-B_{0}\right)$

by Lemma 3.1(iii).

(ii) $\Rightarrow$ (iii). Fix $m, n \in \mathbb{N}$. Let $A_{i}(i=1,2)$ be in $M_{m}(I)$ and $B_{i}(i=1,2)$ be in $M_{n}(I)$. Then

$$
\begin{aligned}
f\left(A_{2}, B_{2}\right)-f\left(A_{1}, B_{1}\right) \geq & f^{[1,0]}\left(A_{1}, B_{1}\right) \circ\left(A_{2}-A_{1}\right) \otimes I \\
& +f^{[0,1]}\left(A_{1}, B_{1}\right) \circ I \otimes\left(B_{2}-B_{1}\right)
\end{aligned}
$$

and

$$
\begin{aligned}
f\left(A_{1}, B_{1}\right)-f\left(A_{2}, B_{2}\right) \geq & f^{[1,0]}\left(A_{2}, B_{2}\right) \circ\left(A_{1}-A_{2}\right) \otimes I \\
& +f^{[0,1]}\left(A_{2}, B_{2}\right) \circ I \otimes\left(B_{1}-B_{2}\right) .
\end{aligned}
$$

On adding the above inequalities, we get the desired result.

(iii) $\Rightarrow$ (i). Let $\varphi:[0,1] \rightarrow M_{m} \otimes M_{n}$ be defined by

$$
\begin{aligned}
\varphi(t) & =f\left(t\left(A_{2}, B_{2}\right)+(1-t)\left(A_{1}, B_{1}\right)\right) \\
& =f\left(t A_{2}+(1-t) A_{1}, t B_{2}+(1-t) B_{1}\right) .
\end{aligned}
$$

Let $t_{1}, t_{2} \in[0,1]$ be such that $0 \leq t_{1}<t_{2} \leq 1$. Set

$$
U_{i}=t_{i} A_{2}+\left(1-t_{i}\right) A_{1} \quad \text { and } \quad V_{i}=t_{i} B_{2}+\left(1-t_{i}\right) B_{1}, \quad i=1,2 .
$$

Then $U_{2}-U_{1}=\left(t_{2}-t_{1}\right)\left(A_{2}-A_{1}\right)$ and $V_{2}-V_{1}=\left(t_{2}-t_{1}\right)\left(B_{2}-B_{1}\right)$. The given condition then implies

$$
\begin{aligned}
& f^{[1,0]}\left(U_{1}, V_{1}\right) \circ\left(A_{2}-A_{1}\right) \otimes I+f^{[0,1]}\left(U_{1}, V_{1}\right) \circ I \otimes\left(B_{2}-B_{1}\right) \\
& \quad \leq f^{[1,0]}\left(U_{2}, V_{2}\right) \circ\left(A_{2}-A_{1}\right) \otimes I+f^{[0,1]}\left(U_{2}, V_{2}\right) \circ I \otimes\left(B_{2}-B_{1}\right) .
\end{aligned}
$$


Observe that

$$
\varphi^{\prime}\left(t_{i}\right)=f^{[1,0]}\left(U_{i}, V_{i}\right) \circ\left(A_{2}-A_{1}\right) \otimes I+f^{[0,1]}\left(U_{i}, V_{i}\right) \circ I \otimes\left(B_{2}-B_{1}\right),
$$

$i=1,2$, by Lemma 3.1(iii). The above inequality then becomes $\varphi^{\prime}\left(t_{1}\right) \leq$ $\varphi^{\prime}\left(t_{2}\right)$, i.e., $\varphi^{\prime}$ is increasing and hence $\varphi$ is convex. Now,

$$
\begin{aligned}
f\left(t\left(A_{2}, B_{2}\right)+(1-t)\left(A_{1}, B_{1}\right)\right) & =\varphi(t)=\varphi(t \cdot 1+(1-t) \cdot 0) \\
& \leq t \varphi(1)+(1-t) \varphi(0) \\
& =t f\left(A_{2}, B_{2}\right)+(1-t) f\left(A_{1}, B_{1}\right) .
\end{aligned}
$$

3.3. TheOREM. Let $f \in C^{2}(I \times I)$, where $I=(-1,1)$, be such that $f(x, 0)=f(0, y)=0$ for $x, y$ in $I$. Then $f$ is operator convex on $I \times I$ iff the matrix

$$
f^{[2,0]}(A, B) \circ H^{2} \otimes I+f^{[1,1]}(A, B) \circ H \otimes K+f^{[0,2]}(A, B) \circ I \otimes K^{2} \geq 0
$$

for $A$ in $M_{m}(I)$ and $B$ in $M_{n}(I)$.

Proof. Let $H \in M_{m}$ and $K \in M_{n}$ be arbitrary. Set $\varphi(t)=f(A+$ $t H, B+t K)$. Then $\varphi(t)$ is a convex function of $t$ in some neighbourhood of the origin. Since $f \in C^{2}(I \times I)$ then so also is $\varphi$, and $\varphi^{\prime \prime}(0) \geq 0$. But

$$
\frac{\varphi^{\prime \prime}(0)}{2 !}=\lim _{t \rightarrow 0} t^{-2}\left\{\varphi(t)-\varphi(0)-t \varphi^{\prime}(0)\right\} .
$$

Also

$$
\begin{aligned}
\varphi^{\prime}(0) & =\lim _{t \rightarrow 0} t^{-1}\{\varphi(t)-\varphi(0)\} \\
& =\lim _{t \rightarrow 0} t^{-1}\{f(A+t H, B+t K)-f(A, B)\} \\
& =f^{[1,0]}(A, B) \circ H \otimes I+f^{[0,1]}(A, B) \circ I \otimes K,
\end{aligned}
$$

by Lemma 3.1(iii). Now,

$$
\begin{aligned}
& t^{-2}\left\{f(A+t H, B+t K)-f(A, B)-t f^{[1,0]}(A, B) \circ H \otimes I-t f^{[0,1]}(A, B) \circ I \otimes K\right\} \\
&= t^{-2}\{f(A+t H, B+t K)-f(A+t H, B)-f(A, B+t K)+f(A, B)\} \\
&+t^{-2}\left\{f(A+t H, B)-f(A, B)-t f^{[1,0]}(A, B) \circ H \otimes I\right\} \\
&+t^{-2}\left\{f(A, B+t K)-f(A, B)-t f^{[0,1]}(A, B) \circ I \otimes K\right\} .
\end{aligned}
$$

Consequently,

$\frac{\varphi^{\prime \prime}(0)}{2 !}=f^{[2,0]}(A, B) \circ H^{2} \otimes I+f^{[1,1]}(A, B) \circ H \otimes K+f^{[0,2]}(A, B) \circ I \otimes K^{2}$.

Thus, if $f$ is operator convex, it then follows that the matrix

$$
f^{[2,0]}(A, B) \circ H^{2} \otimes I+f^{[1,1]}(A, B) \circ H \otimes K+f^{[0,2]}(A, B) \circ I \otimes K^{2}
$$

is non-negative. 
Conversely, if the condition is satisfied then $\varphi(t)$ is convex and hence $f$ is operator convex since its restriction to any line segment in $M_{m}(I) \times M_{n}(I)$ is operator convex.

4. Operator monotonicity of functions of two variables. Lemmas 3.1(iv) and 4.1 provide an alternative proof of a theorem of Korányi [5, Th. 4] and a theorem of Vasudeva [9, Th. 3] - a complete analogue to Löwner's theorem for functions of two variables and its finite-dimensional version respectively.

4.1. Lemma. If $s \rightarrow A(s)$ [resp. $t \rightarrow B(t)]$ is a $C^{1}$ function from $[0,1]$ to the space of $m \times m$ matrices [resp. $n \times n$ matrices] with spectrum in $I=(-1,1)$ and if $f \in C^{1}(I \times I)$, then

$$
\begin{aligned}
f(A(1), B(1))-f & (A(1), B(0))-f(A(0), B(1))+f(A(0), B(0)) \\
= & \int_{0}^{1} \int_{0}^{1} f^{[1,1]}(A(s), B(t)) \circ\left(\frac{d A(s)}{d s} \otimes \frac{d B(t)}{d t}\right) d s d t .
\end{aligned}
$$

Proof. From Lemma 3.1(iv) we observe that

$$
\begin{aligned}
& f\left(A\left(s^{\prime}\right), B\left(t^{\prime}\right)\right)-f\left(A\left(s^{\prime}\right), B(t)\right)-f\left(A(s), B\left(t^{\prime}\right)\right)+f(A(s), B(t)) \\
& \quad=f^{[1,1]}(A(s), B(t)) \circ\left[\left(A\left(s^{\prime}\right)-A(s)\right) \otimes\left(B\left(t^{\prime}\right)-B(t)\right)\right]+\theta\left(s^{\prime}, s, t^{\prime}, t\right),
\end{aligned}
$$

where $\theta\left(s^{\prime}, s, t^{\prime}, t\right) \rightarrow 0$ as $\left|s^{\prime}-s\right| \rightarrow 0,\left|t^{\prime}-t\right| \rightarrow 0$. Choose $\varepsilon>0$. Then there exist integers $m^{\prime}, n^{\prime} \in \mathbb{N}$ such that $\left\|\theta\left(s^{\prime}, s, t^{\prime}, t\right)\right\|<\varepsilon$ whenever $\left|s^{\prime}-s\right|<$ $1 / m^{\prime},\left|t^{\prime}-t\right|<1 / n^{\prime}$. With $A_{k}=A\left(k / m^{\prime}\right), 0 \leq k \leq m^{\prime}$, and $B_{l}=B\left(l / n^{\prime}\right)$, $0 \leq l \leq n^{\prime}$, we therefore have

$$
\begin{gathered}
f(A(1), B(1))-f(A(1), B(0))-f(A(0), B(1))+f(A(0), B(0)) \\
=\sum_{k, l}\left[f\left(A_{k+1}, B_{l+1}\right)-f\left(A_{k+1}, B_{l}\right)-f\left(A_{k}, B_{l+1}\right)+f\left(A_{k}, B_{l}\right)\right] \\
=\sum_{k, l}\left\{f^{[1,1]}\left(A_{k}, B_{k}\right) \circ\left[\left(A_{k+1}-A_{k}\right) \otimes\left(B_{k+1}-B_{k}\right)\right]\right. \\
\left.\quad+\theta\left((k+1) / m^{\prime}, k / m^{\prime},(l+1) / n^{\prime}, l / n^{\prime}\right)\right\} .
\end{gathered}
$$

The first part of the sum converges to the Riemann integral

$$
\int_{0}^{1} \int_{0}^{1} f^{[1,1]}(A(s), B(t)) \circ\left(\frac{d A(s)}{d s} \otimes \frac{d B(t)}{d t}\right) d s d t
$$

as the area of the mesh of the subdivision tends to zero. The second term of the sum is less than the preassigned positive number $\varepsilon$. Hence the result follows. 
4.2. THEOREM. Let $f$ be a real-valued function of two real variables $x$ and $y$ in $I=(-1,1)$. Assume that (i) $f(x, 0)=f(0, y)=0$ for all $x, y$ in $I$ and (ii) the first partial derivatives and the mixed second partial derivative of $f$ exist and are continuous. Then $f$ is a monotone operator function of two variables iff $f^{[1,1]}(A, B) \geq 0$ for every $A \in M_{m}(I)$ and $B \in M_{n}(I)$ and for $m, n \in \mathbb{N}$.

Proof. Fix $m, n \in \mathbb{N}$ and let $A=\sum \lambda_{i} e_{i i}$ and $B=\sum \mu_{i} f_{i i}$, where $\left\{e_{i j}: 1 \leq i, j \leq m\right\}$ and $\left\{f_{i j}: 1 \leq i, j \leq n\right\}$ are matrix units for $M_{m}$ and $M_{n}$ respectively. Suppose $f$ is operator monotone. Choose $H=\sum e_{i j}$ and $K=$ $\sum f_{i j}$. Then $m^{-1} H, n^{-1} K$ are one-dimensional projections. Consequently, $0 \leq \varepsilon_{1}^{-1} \varepsilon_{2}^{-1}\left\{f\left(A+\varepsilon_{1} H, B+\varepsilon_{2} K\right)-f\left(A+\varepsilon_{1} H, B\right)-f\left(A, B+\varepsilon_{2} K\right)+f(A, B)\right\}$, whence $f^{[1,1]}(A, B) \geq 0$, by Lemma $3.1(\mathrm{iv})$, since $H \otimes K$ is the unit for the Hadamard product.

Conversely, suppose that $f^{[1,1]}(A, B) \geq 0$. Choose $A^{\prime} \in M_{m}(I)$ and $B^{\prime} \in M_{n}(I)$ such that $A^{\prime} \geq A$ and $B^{\prime} \geq B$. Set $A(s)=(1-s) A+s A^{\prime}$ and $B(t)=(1-t) B+t B^{\prime}$. Then

Consequently, using Lemma 4.1, we have

$$
\frac{d A(s)}{d s}=A^{\prime}-A \geq 0, \quad \frac{d B(t)}{d t}=B^{\prime}-B \geq 0 .
$$

$$
\begin{aligned}
f\left(A^{\prime}, B^{\prime}\right)- & f\left(A^{\prime}, B\right)-f\left(A, B^{\prime}\right)+f(A, B) \\
& =\int_{0}^{1} \int_{0}^{1} f^{[1,1]}(A(s), B(t)) \circ\left(A^{\prime}-A\right) \otimes\left(B^{\prime}-B\right) d s d t \geq 0,
\end{aligned}
$$

because the Hadamard product of positive matrices is again positive.

Acknowledgements. The authors would like to thank Prof. Ajit Iqbal Singh (née Ajit Kaur Chilana) for useful suggestions.

\section{References}

[1] T. Ando, Topics on Operator Inequalities, lecture notes (mimeographed), Hokkaido University, Sapporo, 1978.

[2] C. Davis, Notions generalizing convexity for functions defined on spaces of matrices, in: Proc. Sympos. Pure Math. 7, Amer. Math. Soc., 1963, 187-201.

[3] W. F. Donoghue, Jr., Monotone Matrix Functions and Analytic Continuation, Springer, Heidelberg, 1974.

[4] F. Hansen and G. K. Pedersen, Jensen's inequality for operators and Löwner's theorem, Math. Ann. 258 (1982), 229-241.

[5] A. Korányi, On a class of analytic functions of several variables, Trans. Amer. Math. Soc. 101 (1961), 521-554.

[6] F. Kraus, Über konvexe Matrixfunktionen, Math. Z. 41 (1936), 18-41.

[7] C. Löwner, Über monotone Matrixfunktionen, ibid. 38 (1934), 177-216. 
[8] A. W. Roberts and D. E. Varberg, Convex Functions, Academic Press, New York, 1973.

[9] H. Vasudeva, On monotone matrix functions of two variables, Trans. Amer. Math. Soc. 176 (1973), 303-318.

DEPARTMENT OF APPLIED MATHEMATICS

REGIONAL ENGINEERING COLLEGE

JALANDHAR, PUNJAB, INDIA
DEPARTMENT OF MATHEMATICS

PANJAB UNIVERSITY CHANDIGARH, INDIA

Reçu par la Rédaction le 20.5.1993 